\title{
Electrochemical sensing of the behaviour of oligonucleotide lipoplexes at charged interfaces
}

\author{
J.A.P. Piedade ${ }^{a}$, M. Mano ${ }^{\text {b,c }}$, M.C. Pedroso de Lima ${ }^{\text {b,c }}$, \\ T.S. Oretskaya ${ }^{\mathrm{d}}$, A.M. Oliveira-Brett ${ }^{\mathrm{a}, *}$

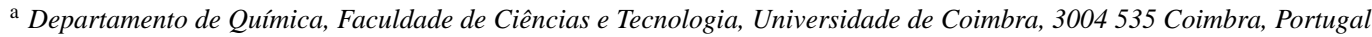 \\ ${ }^{\mathrm{b}}$ Departamento de Bioquímica, Faculdade de Ciências e Tecnologia, Coimbra, Portugal \\ ${ }^{\mathrm{c}}$ Centro de Neurociências e Biologia Celular, Universidade de Coimbra, 3004-535 Coimbra, Portugal \\ ${ }^{\mathrm{d}}$ Chemistry Department, Moscow State University, Vorobjovy Gory, 119899 Moscow, Russia
}

Available online 28 July 2004

\begin{abstract}
Complexes between short oligodeoxynucleotides (ODN) with a variable $\mathrm{dG}_{x} \mathrm{dC}_{y}$ base composition and liposomes composed of the cationic lipid DOTAP (ODN lipoplexes) were studied by differential pulse voltammetry at a glassy carbon electrode. Since lipoplexes are spontaneously formed by electrostatic interactions, the objective of the voltammetric study was to investigate their behaviour at the electrode surface/solution interface. It was verified that the peak current in the voltammograms for ODN lipoplexes was due to guanosine oxidation and that it was influenced both by the applied adsorption potential and the lipoplex $( \pm)$ charge ratio used. It was found that for low ODN lipoplexes $( \pm)$ charge ratios the peak current obtained was enhanced when compared to that registered with free ODN for the same concentration. This allowed a higher sensitivity in the determination of ODN by differential pulse voltammetry and a limit of detection of $5.5 \mathrm{ng} / \mathrm{mL}$ was achieved. A model that explains the organisation of ODN lipoplexes at the electrode surface/solution interface is proposed. The electrochemical results presented account for a better physicochemical characterisation of lipoplexes at charged interfaces, which can be important for the understanding and development of gene therapy vectors based on ODN lipoplexes.
\end{abstract}

(c) 2004 Elsevier B.V. All rights reserved.

Keywords: Voltammetry; Charged interface; Oligonucleotide lipoplex; Cationic liposome; DOTAP

\section{Introduction}

Interactions between lipids and deoxyribonucleic acids (DNA) are gaining increased attention as experimental evidence towards a modulatory role for the interactions between genetic material, phospholipids of biological membranes and the intracellular matrix (Alessenko and Burlakova, 2002; Kuvichkin, 2002).

Although other techniques have also been used for this purpose, lipid/DNA interactions have been extensively studied by electrochemical methods using different approaches and pursuing different objectives (Nikolelis et al., 1999).

Interactions between DNA and lipid bilayer membranes covering electrode surfaces have been used for the development of hybridisation biosensors, in which short oligonu-

\footnotetext{
* Corresponding author. Tel.: +351-239-835295; fax: +351-239-835295.

E-mail address: brett@ci.uc.pt (A.M. Oliveira-Brett).
}

cleotides of known sequences are used as hybridisation probes (Hianik et al., 2003; Siontorou et al., 1996; Wang, 2000).

In addition to their use for detection applications, oligodeoxynucleotides (ODNs) have become the main tool for an antisense based therapeutic approach (Cohen, 1994). Among the strategies that are currently being developed to increase the cellular uptake of ODNs, which are of crucial importance to achieve any therapeutic result, is the use of lipoplexes, complexes formed through spontaneous electrostatic interaction between cationic liposomes and the negatively charged phosphate backbone of DNA. Lipoplexes have been developed and used as a way to deliver DNA (either plasmid DNA or oligonucleotides) into a target cell or tissue aiming the development of appropriate non-viral gene delivery systems for in vitro and in vivo gene therapy (Audory and Hoekstra, 2001; Pedroso de Lima et al., 2001).

The first and still most used cationic lipid in lipoplex formulations is DOTAP (1,2-dioleoyl-3-trimethylammonium 


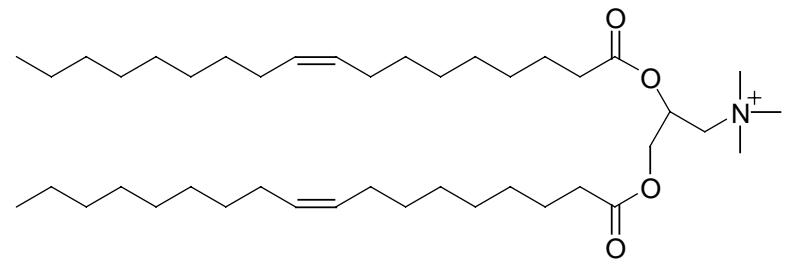

Scheme 1. Molecular structure of DOTAP.

propane), a monovalent positively charged non-natural lipid that exhibits low toxicity in vivo (Scheme 1). DOTAP liposomes have been shown to efficiently mediate the transfection of DNA, RNA and oligonucleotides (Audory and Hoekstra, 2001).

Structural changes both in DNA and liposomes have been shown to occur during lipoplex formation. Circular dichroism and infrared data suggest that double strand DNA is condensed and can suffer various conformational transitions (Choosakoonkriang et al., 2003; Even-Chen and Barenholz, 2000; Prasad et al., 2003; Weimin et al., 2003), while cationic lipids appear to change phase (Bordi et al., 2003; Raedler et al., 1997). When considering ODN lipoplexes, it was recently reported that the nucleotide sequence and composition influence some physicochemical properties of the lipoplexes as the complexation process (Meidan et al., 2001) although a full characterisation of the interaction between ODNs and cationic liposomes is still required (Jurkiewicz et al., 2003; Meidan et al., 2000).

The interaction between lipoplexes and biological membranes is important for the success of gene delivery in to target cells (Pedroso de Lima et al., 2001; Ruponen et al., 2003). It is known that biomembranes have natural transmembrane potentials and the membrane/extracellular interface or intracellular medium can be considered as a charged interface. Several biochemical and physical techniques have been used to characterise lipoplexes (Audory and Hoekstra, 2001), but there is a gap in fundamental bioelectrochemical studies on ODN lipoplexes at charged interfaces. Since lipoplexes are formed due to electrostatic interactions between their components, it is interesting to investigate the influence of the lipoplex $( \pm)$ charge ratio and ODN composition on the stability and structure of ODN lipoplexes when in contact with a charged interface. This can be achieved through voltammetry.

The voltammetry of ODN lipoplexes composed of ODN containing all four DNA bases and DOTAP liposomes has been previously studied by differential pulse voltammetry at a glassy carbon electrode surface (Piedade et al., 2004). It was shown that the ODN base composition and the ODN lipoplex $( \pm)$ charge ratio influenced the voltammetric response confirming the influence of ODN composition on the physicochemical properties of lipoplexes (Meidan et al., 2001).

The main purpose of the present work was to study the behaviour of ODN lipoplexes composed with ODN of vari- able $\mathrm{dG}_{x} \mathrm{dC}_{y}$ base composition and DOTAP liposomes, at the electrode surface/solution interface by differential pulse voltammetry using a glassy carbon electrode.

\section{Materials and methods}

\subsection{Materials}

Analytical grade reagents and purified water from a Millipore Milli-Q system (conductivity $<0.1 \mu \mathrm{S} \mathrm{cm}^{-1}$ ) were used for the preparation of phosphate buffer and electrolyte solution. DOTAP (1,2-dioleoyl-3-trimethylammonium propane) $25 \mathrm{mg} / \mathrm{mL}$ in chloroform solution was purchased from Avanti Polar Lipids (Alabaster, AL) and used without any further purification.

Phosphate buffer solutions, $\mathrm{pH} 7.1$, was chosen in order to work close to the physiologic $\mathrm{pH}$.

The oligodeoxynucleotides used in this study are listed in Table 1 and were synthesised on an Applied Biosystems 380B automated DNA synthesizer (USA) using reagents for oligonucleotide chemistry purchased from Fluka (Germany). Buffered solutions of each ODN were prepared by diluting a certain volume of the ODN aqueous stock solution to get the desired concentration.

Nano- and microvolumes were measured using a EP-10 Plus and a EP-100 Plus Motorized Microliter Pippette (Rainin Instrument Co. Inc., Woburn, USA). The pH measurements were carried out with a Crison Model micro-pH 2001 pH-meter with an Ingold combined glass electrode. All experiments were done at room temperature.

\subsection{ODN quantification and self-dimer formation}

Concentrations of the aqueous solutions of ODNs were determined by measuring their absorbance at $260 \mathrm{~nm}$ using an UV-vis spectrophotometer Specord S 100 (Carl Zeiss Technology, Analytic Jena, Jena, Germany), considering the relation between optical units and $\mu \mathrm{g}$ for each sequence presented in Table 1.

The extent and stability of any possible self-dimers and hairpin structures formed by the ODNs used were evaluated theoretically using the software PrimerSelect (DNASTAR Inc.).

Table 1

Sequence of oligodeoxynucleotide used

\begin{tabular}{lll}
\hline Number & Sequences $\left(5^{\prime} \rightarrow 3^{\prime}\right)$ & Abs 260 (o.u./ $/ \mu \mathrm{g})$ \\
\hline I & GGGGCGGG & 28 \\
II & GGGGCCGGG & 34 \\
III & GGGGCCCGGG & 33 \\
IV & GGGGCCGGGCGGG & 33 \\
V & GGGGCCCGGGCCGGGCGGG & 33 \\
VI & CCCGCCCC & 28 \\
VII & CCCGGGCCCC & 29 \\
\hline
\end{tabular}




\subsection{Preparation of cationic liposomes}

For the preparation of DOTAP (1,2-dioleoyl-3-trimethylammonium propane) liposomes (Simões et al., 1998), a dry film of $3 \mu \mathrm{mol}$ lipid was produced under vacuum using a rotatory evaporator from a stock solution in chloroform. The dried lipid film was then hydrated with distilled water to a lipid concentration of $3 \mu \mathrm{mol} / \mathrm{mL}$ and sonicated for $5 \mathrm{~min}$. The resulting liposomes were then extruded 21 times through two stacked polycarbonate membranes of $50 \mathrm{~nm}$ pore diameter using a Liposofast device (Avestin, Toronto, Canada), and stored at $4{ }^{\circ} \mathrm{C}$, under nitrogen, until use. Liposomes were used within 1 month after preparation.

\subsection{Lipoplex formation}

The complexes between DOTAP liposomes and ODN (ODN lipoplexes) were prepared at different DOTAP/ODN $( \pm)$ charge ratios by gently mixing the phosphate buffered ODN solution with a fixed volume of the liposome stock solution, depending on the charge ratio desired. The mixture was left for $15 \mathrm{~min}$ at room temperature prior to any voltammetric experiment.

In the present work, the molar $( \pm)$ charge ratio of DOTAP to negatively charged phosphates in ODN was varied between 0 and $2 / 1$, and was calculated considering that $1 \mu \mathrm{mol}$ of cationic lipids corresponds to $1 \mu \mathrm{mol}$ of positive charges and $1 \mathrm{mg}$ of DNA has $3.08 \mathrm{nmol}$ of negatively charged phosphate groups at $\mathrm{pH}$ 7.1. The final concentrations of lipid used to generate the ODN lipoplex molar charge ratio desired ranged from 0.06 and $0.9 \mathrm{mmol} / \mathrm{L}$, that is in the range of lipid concentrations normally used in gene therapy formulations (Audory and Hoekstra, 2001; Pedroso de Lima et al., 2001; Simões et al., 1998).

\subsection{Voltammetric cell and parameters}

All voltammetric experiments were done using an $\mu$ Autolab Type II potentiostatrunning with GPES version 4.9 software (Eco-Chemie, Utrecht, The Netherlands). A one-compartment electrochemical cell with a volumetric capacity of $100 \mu \mathrm{L}$ was used containing a $1.5 \mathrm{~mm}$ diameter glassy carbon electrode (GCE) (Cypress, USA), a Pt wire counter electrode, and an $\mathrm{Ag} / \mathrm{AgCl}$ reference electrode (the electrode filling solution was $\mathrm{KCl} 3 \mathrm{M}$ saturated with $\mathrm{AgCl})$. This cell was placed inside a Faraday cage at room temperature during all the measurements.

The voltammetric parameters used, unless stated otherwise, were: differential pulse voltammetry, pulse amplitude $50 \mathrm{mV}$, pulse width $70 \mathrm{~ms}$, scan rate $5 \mathrm{mV} \mathrm{s}^{-1}$, equilibrium time $10 \mathrm{~s}$. All potentials are referred to $\mathrm{Ag} / \mathrm{AgCl}$.

\subsection{Pre-conditioning of the glassy carbon mini-electrode}

Before and between every electrochemical assay with samples of ODNs the glassy carbon electrode was polished using alumina oxide, particle size $0.3 \mu \mathrm{m}$. After polishing, the electrode was rinsed thoroughly with Milli-Q water during $30 \mathrm{~s}$, sonicated for $1 \mathrm{~min}$ in a ultrasound bath, and again rinsed with water. After this mechanical treatment the electrode received an electrochemical pre-conditioning that consisted of three steps: first, the electrode was scanned in the electrolyte solution to check the cleanness of the electrode surface; second, a potential of $+1.65 \mathrm{~V}$ versus $\mathrm{Ag} / \mathrm{AgCl}$ was applied for $300 \mathrm{~s}$; and finally the electrode was scanned between +0.30 and $+1.30 \mathrm{~V}$, until a steady-state base line voltammogram was obtained. This electrochemical pre-conditioning improves the faradaic currents recorded making the assay more sensitive and reproducible (Hong-Ping and Kwok-Keung, 1996; Piedade et al., 2004), and so this pre-conditioning of the electrode surface was employed in all the voltammetric experiments presented.

\subsection{Acquisition and presentation of voltammetric data}

After the electrochemical pre-conditioning the GCE was immersed in the sample solution and applied a chosen adsorption potential (varying between -0.30 and $+0.40 \mathrm{~V}$ ) was applied for $300 \mathrm{~s}$. The GCE with the adsorbed sample was then gently washed with deionised water and transferred to a phosphate buffer supporting electrolyte solution. The voltammograms were recorded between +0.30 and $+1.30 \mathrm{~V}$ versus $\mathrm{Ag} / \mathrm{AgCl}$.

All the experimental curves presented were backgroundsubtracted and base line corrected using the moving average application with a step window of $10 \mathrm{mV}$ included in GPES version 4.9 software. This mathematical treatment improves the visualisation and identification of peaks over the base line without introducing any artefact, although the peak intensity is in some cases reduced $(<10 \%)$ relative to that of the raw curve. The values for peak current presented in all graphs were determined from the original raw voltammograms.

\section{Results}

It has been shown recently (Piedade et al., 2004) that the ODN base composition and the ODN lipoplex $( \pm)$ charge ratio influenced the voltammetric response obtained. Therefore, in order to investigate the behaviour of ODN lipoplexes at charged interfaces and to avoid complications due to base sequence effects on the voltammetric response, ODN sequences containing only guanine $(\mathrm{G})$ and cytosine (C) bases (Table 1) were used in the present work.

\subsection{Voltammetry of ODN, DOTAP liposomes and ODN lipoplexes}

The differential pulse voltammograms obtained for the free ODN-I, DOTAP liposome suspension, and for the ODN-I lipoplex with a $( \pm)$ charge ratio of $1 / 1$, are presented in Fig. 1. 
The voltammogram obtained for ODN-I presents a single peak current at $+0.90 \mathrm{~V}$, Fig. 1 (full line), corresponding to the oxidation of guanosine present in the ODN (Oliveira-Brett et al., 1999; Piedade et al., 2004), which will be referred to as peak-dG. The peak current is proportional to the concentration of the guanosine present in the ODN and it also reflects the accessibility of the guanine nucleotides in the ODN to the surface of the electrode since their adsorption is essential for charge transfer to occur (Oliveira-Brett et al., 1999; Palecek et al., 2002). As expected (Oliveira-Brett and Matysik, 1997; Piedade et al., 2004), the oxidation of the cytosine nucleotides in the ODN was not seen because cytidine can be only detected at high positive potential, for the $\mathrm{pH}$ used, and its oxidation peak is usually masked by oxidation of the electrolyte solution.

Only one peak current was detected for ODN-I lipoplexes with a $( \pm)$ charge ratio of $1 / 1$, at the same potential at which the oxidation of guanosine in the free ODN-I was observed, with a small but not significant current decrease, Fig. 1 (dashed line).

It was verified that the DOTAP liposome suspension voltammogram shows no faradaic response, Fig. 1 (dotted line) when the potential was scanned between -0.30 and $+1.40 \mathrm{~V}$, indicating that DOTAP does not suffer any electro-oxidation or reduction reaction in this working potential window and for the $\mathrm{pH}$ used. Therefore, the peak observed for the ODN-I lipoplex was considered to be due only to the oxidation of guanine nucleotides in the lipoplex adsorbed onto the GCE surface. Since no other peaks were detected, peak-dG was considered to be a convenient ana-

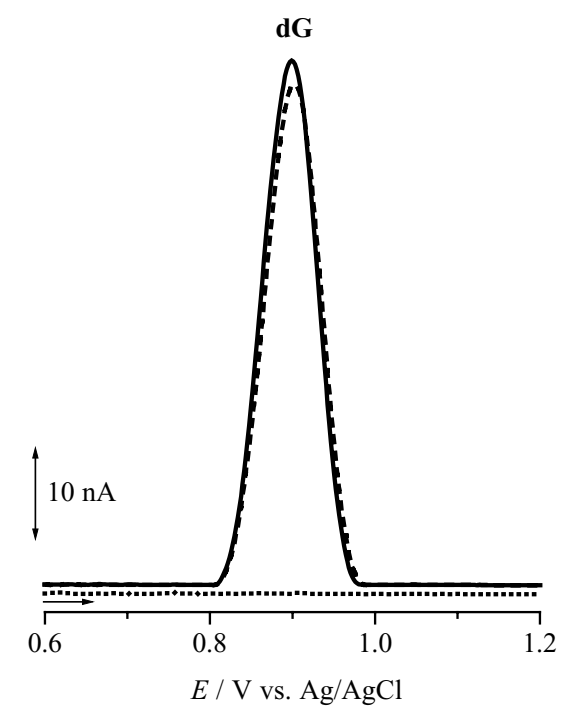

Fig. 1. Base line corrected differential pulse voltammograms obtained in $0.1 \mathrm{M}$ phosphate buffer ( $\mathrm{pH} 7.1)$ for solutions of: $20 \mathrm{nmol} / \mathrm{mL}(56 \mu \mathrm{g} / \mathrm{mL})$ of ODN-I (full line), $172 \mathrm{nmol} / \mathrm{mL}$ of DOTAP liposome suspension (dashed line); ODN-I lipoplex of $( \pm$ ) charge ratio 1/1 (dotted line). Adsorption at $-0.10 \mathrm{~V}$ for $300 \mathrm{~s}$ was carried out before transferring the electrode for measurement in electrolyte solution. Pulse amplitude $50 \mathrm{mV}$, pulse width $70 \mathrm{~ms}$, scan rate $5 \mathrm{mV} \mathrm{s}^{-1}$; dG: peak due to guanosine oxidation. lytical peak for studying the voltammetric behaviour of this group of ODN lipoplexes at the electrode surface/solution interface.

\subsection{Effect of ODN lipoplexes charge ratio and adsorption potential on peak current}

The effect of the ODN lipoplex $( \pm)$ charge ratio and the adsorption potential on the peak-dG current was studied. It was found that the application of a potential where adsorption occurs equal to $-0.10 \mathrm{~V}$ during $300 \mathrm{~s}$ causes a significant increase in peak-dG current for all the ODN-I lipoplex $( \pm)$ charge ratios studied, compared to the case where no potential was applied but the electrode remained immersed in the lipoplex solution for the same period of time, Fig. 2.

The effect of the application of other different potentials and different ODN-I lipoplex $( \pm)$ charge ratios on peak-dG current is shown in the 3D plot in Fig. 3. The results obtained for an adsorption potential of $-0.10 \mathrm{~V}$, already presented in Fig. 2, are shown with the points obtained for different lipoplex $( \pm)$ charge ratios linked with the bolded line in other to facilitate comparison. The applied potential was varied between -0.40 and $+0.30 \mathrm{~V}$ for two reasons: the lower limit was chosen to be $-0.40 \mathrm{~V}$ to avoid oxygen reduction that could otherwise interfere with the results since the voltammetric experiments were done under normal atmosphere; the upper limit was fixed at $+0.30 \mathrm{~V}$ to avoid any undesired electrochemical oxidative damage to the ODN before the voltammetric scan (Oliveira-Brett et al., 1999; Piedade et al., 2004).

It was observed, as a general trend, that the application of a less positive adsorption potential caused an increase in peak-dG current. This effect was observed also for the free ODN-I (charge ratio 0) and can be explained consid-

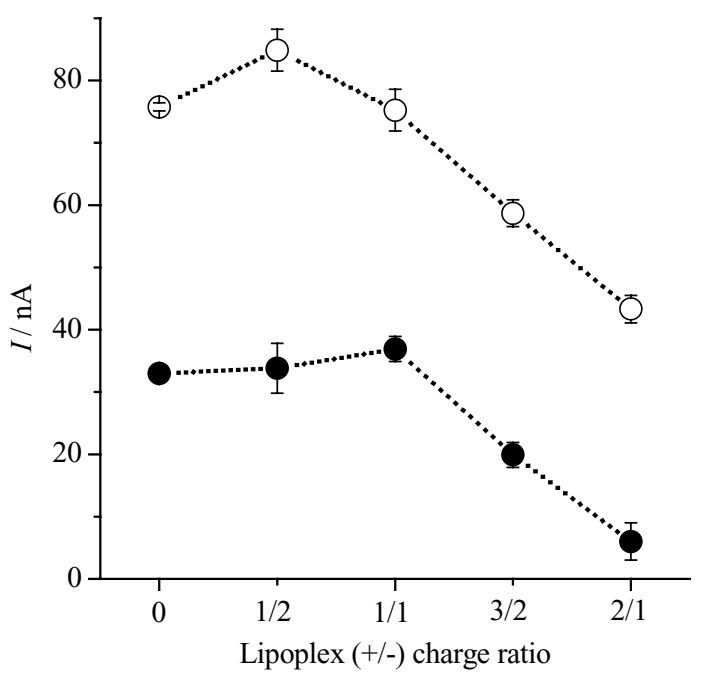

Fig. 2. Curve for of $I$ vs. lipoplex $( \pm)$ charge ratio obtained with (open circle) or without (full circle) the application of an adsorption potential of $-0.10 \mathrm{~V}$ during $300 \mathrm{~s}$. Each error bar represents the standard deviation obtained for three experiments. Experimental conditions as in Fig. 1. 


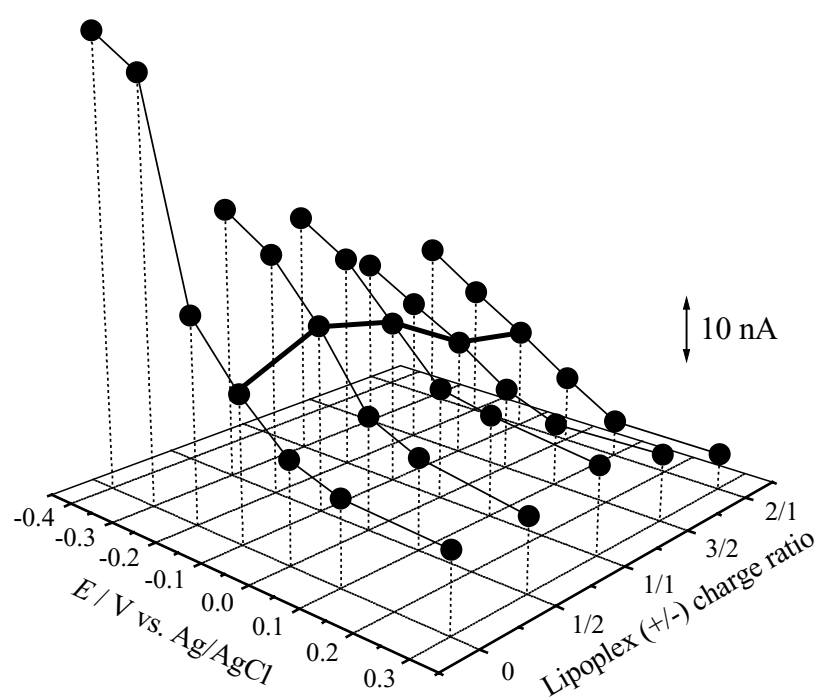

Fig. 3. 3D plot for peak-dG current $(I)$ as function of the adsorption potential $(E)$ and lipoplex $( \pm)$ charge ratio. The black bold line links the points obtained with the application of an adsorption potential of $-0.10 \mathrm{~V}$, Fig. 2. Each point represents the mean value found for three experiments. For experimental details, see text.

ering two contributions. First, the application of a negative adsorption potential leads the ODN to adsorb preferentially with the bases facing the electrode surface due to an increased electrostatic repulsion over the negative backbone phosphate groups. In this case, interaction between the bases and the hydroxyl and carboxylic groups present on GCE surface (Hong-Ping and Kwok-Keung, 1996) are facilitated by hydrogen bounds, increasing the amount of ODN that remains adsorbed at the electrode surface. Second, with guanine base, already close to the electrode surface, it was expected that charge transfer would be facilitated, observed as an increase in the peak-dG current. The tendency of the peak-dG current to increase diminishes with the application of a potential more negative than $-0.30 \mathrm{~V}$, as can be seen in Fig. 3. This agrees well with the above explanation, since it was expected that at more negative adsorption potentials the electrostatic repulsion between the negatively charged phosphate backbone and the electrode surface would predominate over other possible interactions with the solid electrode surface.

In general, the same shape as in Fig. 2 was observed in the curves obtained for peak-dG current versus lipoplex $( \pm)$ charge ratio for adsorption potentials more positive than $-0.20 \mathrm{~V}$. The peak-dG current increased significantly for a lipoplex $( \pm)$ charge ratio of $1 / 2$ in relation to the value obtained for free ODN (charge ratio 0 ). This increase was more accentuated at an applied potential of $-0.20 \mathrm{~V}$, diminishing slightly at more positive potentials. However, the value of peak-dG current although decreasing with lipoplex $( \pm)$ charge ratio, never reached zero even for higher charge ratios. In fact, it was observed that peak-dG current remained almost constant for ODN lipoplexes of $( \pm)$ charge ratios between $2 / 1$ and $4 / 1$ (data not shown). These results demon- strate that the electrode surface was not blocked by DOTAP liposomes, even when a larger excess of liposomes was used.

In order to investigate the interfacial organisation of the adsorbed ODN lipoplex, five different experiments were performed in which different experimental adsorption conditions were chose using ODN-I, liposomes or ODN-I lipoplex solutions. Adsorption at $-0.10 \mathrm{~V}$ for $300 \mathrm{~s}$ was carried out before transferring the electrode for measurement in electrolyte solution. Values of peak-dG current were obtained by differential pulse voltammetry in $0.1 \mathrm{M}$ phosphate buffer $(\mathrm{pH}$ 7.1) electrolyte solution for free ODN-I (bar 1); free ODN-I after a further $300 \mathrm{~s}$ application of a potential of $-0.10 \mathrm{~V}$ in supporting electrolyte (bar 2); DOTAP/ODN lipoplex $( \pm$ ) charge ratio 1/1 (bar 3); DOTAP followed by free ODN-I (bar 4); and free ODN-I followed by DOTAP (bar 5). The values obtained in each experiment for peak-dG current are in the bar chart presented in Fig. 4. Each error bar represents the standard deviation obtained for three experiments.

No significant difference was found between the two first cases (bars 1 and 2), respectively, where the electrode was immersed in the ODN-I solution a potential of $-0.10 \mathrm{~V}$ was applied for $300 \mathrm{~s}$ before transferring the electrode for measurement in electrolyte solution (bar 1), the difference being that after the electrode had been transferred to the electrolyte solution in the second case a potential of $-0.10 \mathrm{~V}$ was again applied for $300 \mathrm{~s}$ before the voltammetric scan (bar 2). The similarity of the results was as expected, as there was no ODN in the bulk electrolyte solution to diffuse and adsorb onto the electrode surface. It also means that application of a second adsorption period in the electrolyte solution does not cause any rearrangement in the ODNs adsorbed at the electrode surface that could eventually cause a variation in

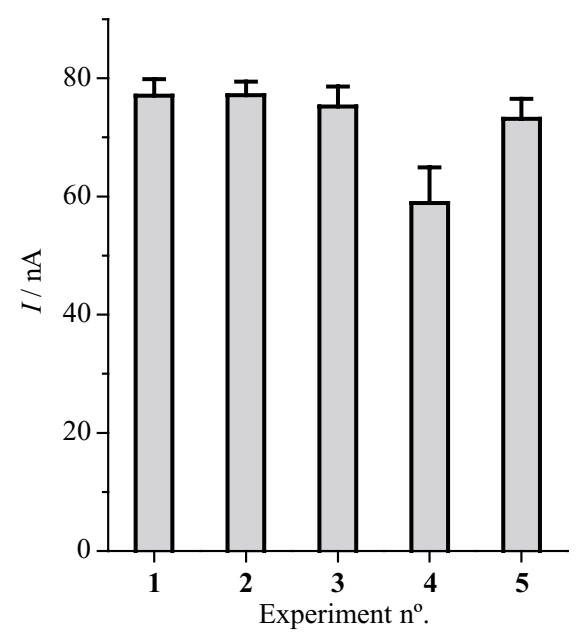

Fig. 4. Values of peak-dG current obtained by differential pulse voltammetry in $0.1 \mathrm{M}$ phosphate buffer ( $\mathrm{pH} 7.1$ ) electrolyte solution for: (1) free ODN-I; (2) free ODN-I with an extra time of adsorption; (3) DOTAP/ODN lipoplex $( \pm$ ) charge ratio $1 / 1$; (4) DOTAP + free ODN-I; (5) free ODN-I + DOTAP. Adsorption at $-0.1 \mathrm{~V}$ for $300 \mathrm{~s}$ was carried out before transferring the electrode for measurement in electrolyte solution. See text for other experimental details. Each error bar represents the standard deviation obtained for three experiments. 
the peak-dG current. These two experiments (bars 1 and 2) were important as controls for the following experiments.

A slight decrease in peak-dG current occurred for ODN-I lipoplexes prepared at a 1/1 $( \pm)$ charge ratio and adsorbed at $-0.10 \mathrm{~V}$ for $300 \mathrm{~s}$ before transferring the electrode for measurement in electrolyte solution (bar 3) showing as before, that almost all the ODN-I in the lipoplex was still available and accessible to oxidation at the electrode surface.

In the fourth experiment (bar 4), the electrode was first immersed in a DOTAP liposome solution, a potential of $-0.10 \mathrm{~V}$ applied during $300 \mathrm{~s}$, the electrode transferred to the ODN-I solution and the same adsorption procedure repeated. Finally, the electrode was gently washed and transferred to electrolyte solution and the voltammetric scan was performed. In the fifth experiment (bar 5), the order in experiment 4 was inverted: the first period of adsorption was in the ODN-I solution and the second period in the liposome solution. The values of peak-dG current found in experiments 4 and 5 were lower than those registered in the experiment with the ODN-I lipoplex (bar 3) but a larger decrease was observed in experiment 4 . This result can be explained by considering that when the lipid was allowed to adsorb first (experiment 4), the total area of the electrode surface that remained uncovered by liposomes diminished, and consequently less free ODN would be able to adsorb on its surface and be oxidised.

The result in experiment 5 showed that adsorbed ODN-I can interact with the DOTAP liposome solution leading to the formation of some lipoplexes that will decrease the ODN-I free that can be oxidised, either by decreasing its accessibility to the electrode surface or due to desorption and consequent diffusion to bulk electrolyte solution.

In conclusion, this set of five experiments clearly showed that the ODN lipoplexes present distinct voltammetric responses compared to those obtained when free ODN and cationic liposomes interact sequentially at the GCE surface.

\subsection{Effect of ODN length and guanine content on peak current}

The effect on the voltammetric behaviour caused by the percentual content in guanine (ratio G/C) and the length of the ODN used to prepare the ODN lipoplexes was studied using the sequences listed in Table 1. The quantities of each ODN used were calculated for each case to a final concentration of $20 \mathrm{nmol} / \mathrm{mL}$. The amount of lipid added in each case to form the lipoplexes was calculated to obtain the desired molar $( \pm)$ charge ratio within the interval studied $(0-2 / 1)$. The results obtained are shown in Fig. 5.

It was found that the variation of peak-dG current with the lipoplex $( \pm)$ charge ratio follows a similar profile independently of the ODN used, although each ODN presents different peak-dG current values.

It was observed that for sequences with the same content in guanine (ODNs-I-III) the peak-dG current increased with increasing number of cytosine nucleotides for all the lipoplex

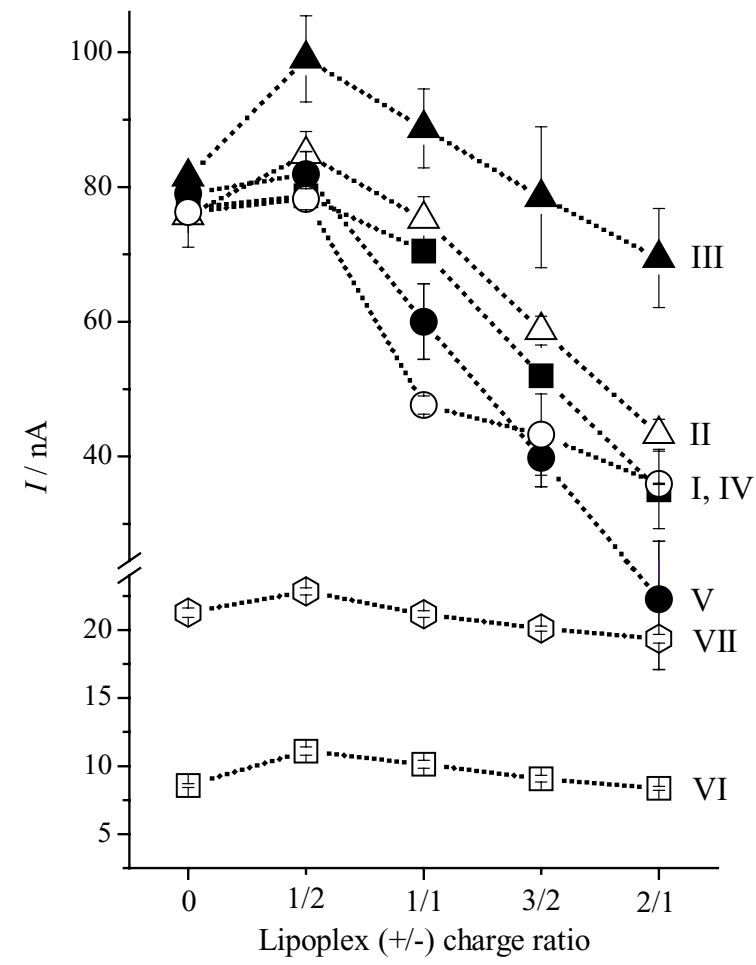

Fig. 5. Experimental relation found for peak-dG current $(I)$ vs. lipoplex $( \pm)$ charge ratio for different ODN sequences: ODN-I (black square); ODN-II (white triangle); ODN-III (black triangle); ODN-IV (white circle); ODN-V (black circle); ODN-VI (white square); ODN-VII (white hexagon). Each error bar represents the standard deviation obtained for three experiments. Experimental conditions as in Fig. 1.

$( \pm)$ charge ratios studied. This was explained considering that the rise in the number of negative charges per oligonucleotide leads to a greater interaction with cationic liposomes causing the formation of some ODN lipoplex aggregates (clusters), which finally results in a higher number of ODNs being transported to the electrode surface. Consequently, a higher number of guanine residues can undergo oxidation which is consistent with the observed increase in peak-dG current. A similar behaviour was observed when ODNs containing bases other than $\mathrm{G}$ and $\mathrm{C}$ were used (Piedade et al., 2004).

For ODN-IV and ODN-V lipoplexes, comparably lower peak-dG current were observed and these results were apparently unexpected since the content in guanine of both sequences was higher than those present in previous sequences.

It is well known that the electrochemical signals due to base oxidation are depressed or even disappear in double stranded DNA (Oliveira-Brett et al., 1999) and the results obtained can be explained considering the formation of self-dimers or hairpins. In fact, the analysis of the sequences of the ODNs for their tendency to form self-dimers and hairpins predicted the formation of 11 self-dimers and 13 hairpins (three of them stable) structures for ODN-V, and of six self-dimers and three hairpins for ODN-IV, while for ODNs-I-III none of these structures were predicted to be 
formed. The fact that the values for peak-dG current obtained for ODN-IV become bigger than those for ODN-V at charge ratios higher than $3 / 2$ and equal to the peak-dG current registered for the ODN-I lipoplex of $( \pm)$ charge ratio $2 / 1$, was explained considering that the increase in lipid content causes the linearisation of the secondary structures predicted for that sequence, in this way allowing more guanine nucleotides to be exposed to the electrode surface and to be able to undergo oxidation. This is in accordance with other results that referred that lipoplex formation can cause changes to DNA conformation and even its denaturation (Choosakoonkriang et al., 2003; Prasad et al., 2003).

Fig. 5 also shows the results obtained for ODN-VI and ODN-VII in which the number of cytosines is constant but the guanine nucleotides triplicate. In this case, the higher values of peak-dG current registered with ODN-VII were explained by the fact that ODN-VII has three times more content in guanine than ODN-VI (Table 1) and the fact that the number of negative charges per ODN also increases. Again the tendency of the curve of peak-dG current versus lipoplex $( \pm)$ charge ratio was similar for both sequences.

The relation between the calculated slope for the curves of peak-dG current versus lipoplex $( \pm$ ) charge ratio (between 1/2 and 2/1) in Fig. 5 and the guanine content in each ODN sequence is presented in Fig. 6. A linear dependence was found between those two variables which implies that the higher the content in guanine in relation to cytosine in a particular ODN, the bigger the decrease in the peak-dG current observed for guanine nucleotide oxidation as the ODN lipoplex $( \pm)$ charge ratio is increased. This means that increasing the number of cytosines will stabilise the ODN lipoplex making the oxidation of $\mathrm{G}$ residues difficult, which is in agreement with previous results (Piedade et al., 2004).

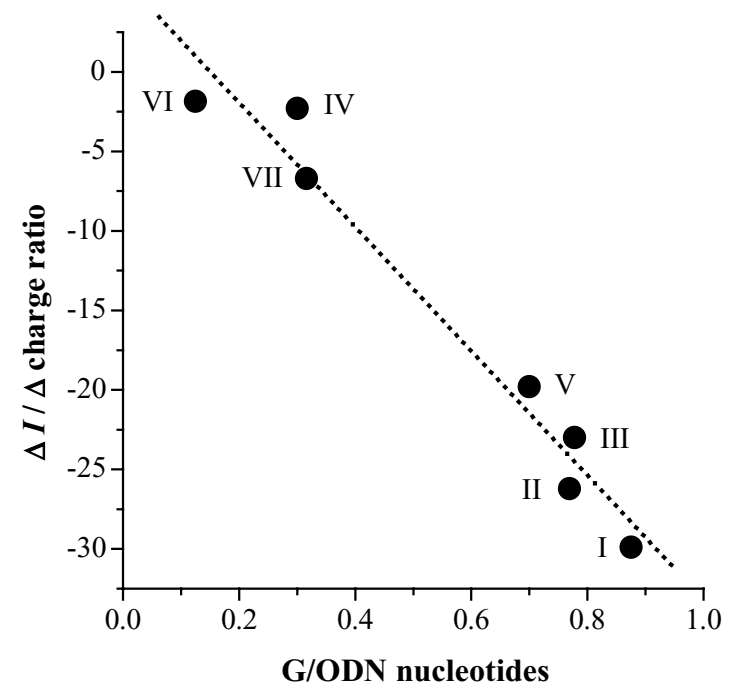

Fig. 6. Relation between $\{\Delta I /(\Delta$ lipoplex charge ratio $)\}$, the change of peak-dG current with change in lipoplex charge ratio, with ODN content in guanine. Data obtained from the experiments presented in Fig. 5.

\subsection{Limit of detection of ODN in lipoplexes by voltammetry}

The results obtained with all ODN lipoplexes studied showed that the peak-dG current was maximum when a lipoplex $( \pm$ ) charge ratio of $1 / 2$ was used and that this value was always higher than that obtained with the free ODN, Figs. 2, 3, 5 and 7. This peak current enhancement caused by the addition of small amounts of liposomes to an ODN solution was used to verify the lipoplex transport effect on

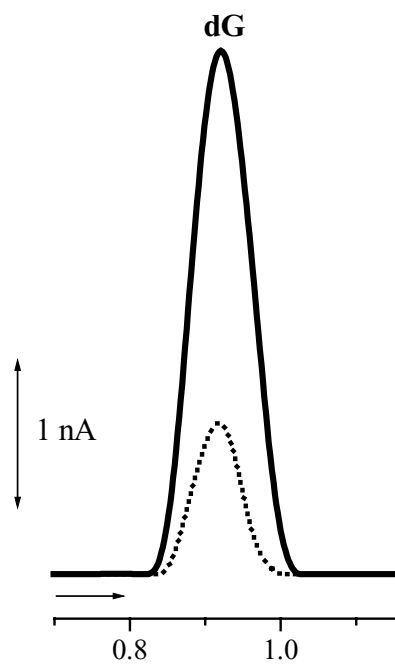

(A) $E / \mathrm{V}$ vs. $\mathrm{Ag} / \mathrm{AgCl}$

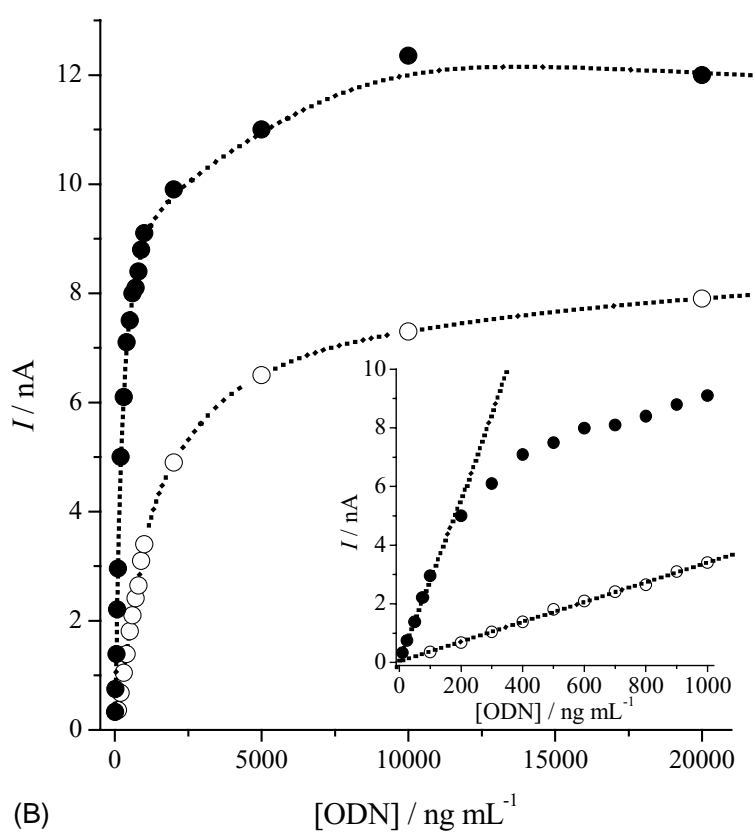

Fig. 7. (A) Base line corrected differential pulse voltammograms obtained in $0.1 \mathrm{M}$ phosphate buffer ( $\mathrm{pH} 7.1$ ) electrolyte solution for $100 \mathrm{ng} / \mathrm{mL}$ of free ODN-VI (doted line) or in lipoplex of $( \pm$ ) charge ratio $1 / 2$ (full line). Adsorption at $-0.10 \mathrm{~V}$ for $300 \mathrm{~s}$ was carried out in the lipoplex solution before transferring the electrode for measurement in the electrolyte solution. Pulse amplitude $50 \mathrm{mV}$, pulse width $70 \mathrm{~ms}$, scan rate $5 \mathrm{mV} \mathrm{s}^{-1}$. (B) Plots obtained for $I$ vs. ODN-VI concentration: ODN-VI lipoplex of $( \pm)$ charge ratio 1/2 (black circle); free ODN-VI (white circle). 
the ODN limit of detection (LOD). Hence, the relation between peak-dG current and ODN concentration using free ODN-VI or ODN-VI lipoplexes prepared at a $( \pm)$ charge ratio of $1 / 2$ was studied and the limit of detection (LOD) for each case determined.

Fig. 7A shows differential pulse voltammograms obtained for $100 \mathrm{ng} / \mathrm{mL}$ of ODN-VI and for the correspondent ODN-VI lipoplex with a $( \pm)$ charge ratio of $1 / 2$. As can be seen, the peak-dG current registered for the ODN lipoplex was about three times higher than that obtained for the free ODN.

The variation of peak-dG current versus ODN-VI concentration obtained for both cases is shown in Fig. 7B. Although the two curves follow the same tendency, it was observed that peak-dG current values obtained in the ODN lipoplex solution were always higher than the values found in the free ODN solution, in the concentration range studied $(10-20000 \mathrm{ng} / \mathrm{mL})$. A significant reduction of about one order of magnitude in the LOD for the ODN was also found when lipoplexes were used, as shown in the inset of Fig. 7B. The LOD obtained by differential pulse voltammetry for the free ODN was $48 \mathrm{ng} / \mathrm{mL}$ ( $2 \mathrm{nmol})$, based on three times the noise level (Brett and Oliveira-Brett, 1998), with a good linearity between 100 and $1000 \mathrm{ng} / \mathrm{mL}$, described by the equation $I(\mathrm{nA})=0.041+0.003[\mathrm{ODN}]\left(\mathrm{ng} \mathrm{mL}^{-1}\right)(R$ $=0.9990 ;$ S.D. $=0.048 ; N=10$ ). For the ODN lipoplexes a lower LOD of $5.5 \mathrm{ng} / \mathrm{mL}$ ( $240 \mathrm{fmol})$ was achieved, with a good linear response between peak-dG current and concentration in the range from 10 to $200 \mathrm{ng} / \mathrm{mL}$ described by the equation $I(\mathrm{nA})=0.006+0.029[\mathrm{ODN}]\left(\mathrm{ng} \mathrm{mL}^{-1}\right)$ $(R=0.9991 ;$ S.D. $=0.053 ; N=6)$. The higher sensitivity achieved with lipoplexes was found to be highly reproducible with a R.S.D. of $4 \%$ found in five determinations of $100 \mathrm{ng} / \mathrm{mL}$ of ODN-VI in the lipoplex.

The ODN-VI was chosen because it contains only one guanine nucleotide so sequences with more guanine nucleotides can be determined more easily.

Finally, the fact that the peak-dG current remains almost constant for ODN concentrations higher than $10 \mu \mathrm{g} / \mathrm{mL}$, Fig. 7B, indicates that all the experiments presented in this work were done close to full electrode surface coverage by the ODN lipoplexes (ODN concentrations in Figs. 1-5 was equal to $56 \mu \mathrm{g} / \mathrm{mL}$ ).

\section{Discussion}

It was shown that it is possible to determine by voltammetric techniques the effect of the ODN lipoplex $( \pm)$ charge ratio on the amount of ODN that is adsorbed at the electrode surface where it can undergo oxidation, either by reorganisation of the ODN lipoplexes at the electrode surface/solution interface or by the dissociation mechanism of ODNs from the lipoplexes during a positive voltammetric scan. A model that explains these events at the electrode surface/solution interface is of interest and considering biological membranes as charged interfaces, it can give insights into the dissociation of ODNs from lipoplexes at the biological level, an important event for the success of ODN delivery to target cells (Audory and Hoekstra, 2001; Pedroso de Lima et al., 2001; Ruponen et al., 2003).

\subsection{Model for ODN lipoplexes behaviour at the electrode/solution interface}

Based on the previous work (Piedade et al., 2004) and experimental results presented here and taking into account other physicochemical properties and the structure of the lipoplexes in general (Bordi et al., 2003; Choosakoonkriang et al., 2003; Dunlap et al., 1997; Even-Chen and Barenholz, 2000; Prasad et al., 2003; Raedler et al., 1997; Weimin et al., 2003; Wiethoff et al., 2002) and of the ODN lipoplexes in particular (Jurkiewicz et al., 2003; Meidan et al., 2000, 2001), a model was proposed for the behaviour of ODN lipoplexes at the electrode surface/solution interface and is presented in Scheme 2. The model is applicable to all the ODN lipoplex $( \pm)$ molar charge ratios studied and the two cases, for low and high charge ratios, are discussed here.

In the case when lipoplexes are formed at charge ratios lower than 1/1, Scheme 2I, the formation of large aggregates of lipoplexes is not expected (Meidan et al., 2001) and the population of lipoplexes will be mainly formed by unitary liposome covered with several ODNs (Jurkiewicz et al., 2003). When the GCE is immersed in the sample solution, ODN lipoplexes diffuse from the bulk solution to the electrode surface (Ia). The application of an adsorption potential of $-0.10 \mathrm{~V}$ changes the charge of the electrode surface and the adsorption of ODN lipoplexes are preferred over free ODN adsorption due to electrostatic interactions (Ib). Some level of reorganisation in the adsorbed ODN lipoplexes is assumed to occur and this reorganisation increases progressively during the positive voltammetric scan (between +0.30 and $+1.30 \mathrm{~V}$ ), due to the increase in the positive charge of the electrode surface (Ic). This interface charge change leads to electrostatic attraction of the ODN and repulsion of the cationic lipids that compose the lipoplexes, originating two main layers (probably not completely homogeneous) at the electrode surface/solution interface. During the positive scan, but before the oxidation potential of peak-dG $(\approx+0.90 \mathrm{~V})$, presumably all ODN remains sandwiched between the electrode surface and the liposomes. At the end of the positive voltammetric scan (Id) almost all lipoplexes have eventually been disrupted and, in this configuration, neighbouring liposomes can fuse giving rise to a lipid bilamellar phase (Meidan et al., 2000; Monkkonen and Urtti, 1998).

In the case that the ODN lipoplexes are prepared at $( \pm)$ charge ratio higher than $1 / 1$, Scheme 2II, clusters of the ODN lipoplex aggregates are expected to be formed and the size of each cluster is supposed to increase with charge ratio (Meidan et al., 2000; Wiethoff et al., 2002). As in the previous case, when the electrode is immersed in the 
I

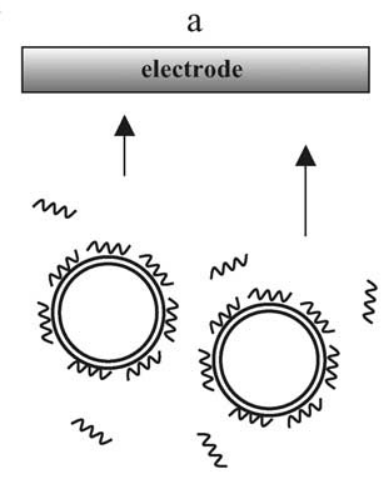

II

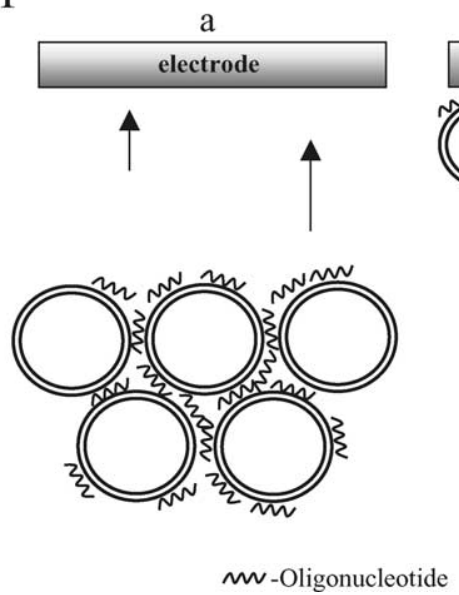

b
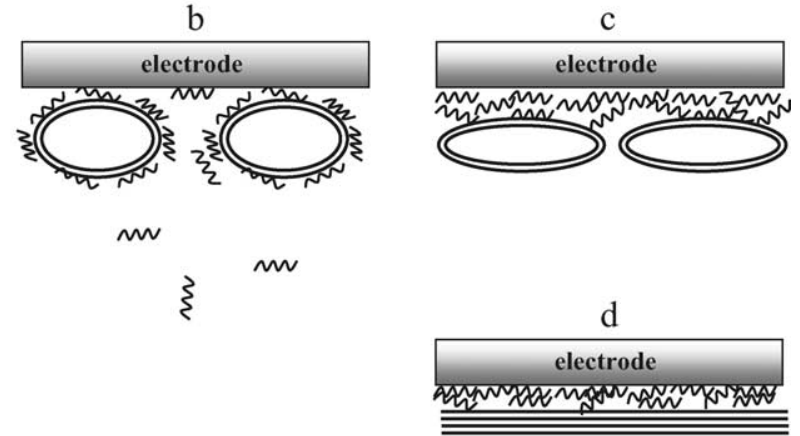
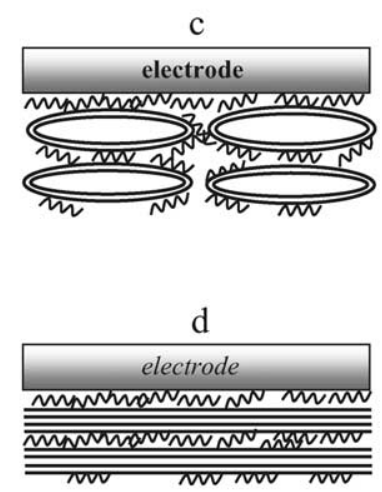

= - DOTAP bilayer

Scheme 2. Proposed model for layer organisation of the ODN lipoplexes at the electrode/solution charged interface. Low (I) and high (II) lipoplex molar charge ratio situations are presented as examples. (a) Formation of ODN lipoplexes and diffusion onto the electrode surface; (b) application of an adsorption potential of $-0.10 \mathrm{~V}$ during $300 \mathrm{~s}$; (c) reorganisation of ODN lipoplexes during positive potential scan between +0.30 and $+1.30 \mathrm{~V}$ in electrolyte solution; (d) lateral fusion of neighbouring liposomes.

sample solution the ODN lipoplex clusters diffuse from the bulk solution and adsorb onto the electrode surface (IIa). But because of the expected greater ODN lipoplex clusters, not all of the transported ODN will be close enough to come into contact with the electrode surface during both the adsorption procedure (IIb) and the positive voltammetric scan. In this case the ODN lipoplex reorganisation creates multi-heterogeneous layers (IIc). A significant portion of ODN stays in layers not in direct contact with the electrode surface (IId) and this physical limitation is responsible for the decrease of peak-dG current observed for higher lipoplex charge ratios (Figs. 2, 3 and 5).

\section{Conclusions}

Besides the application of ODN lipoplexes to improve the sensitivity of the electrochemical detection of ODNs (Fig. 7), it is possible to extend the applicability of electroanalytical methods to study important parameters of lipoplexes used for gene therapy purposes, as is the quantification of the amount of genetic material protected or released by the lipoplex formulation when in contact with a charged interface.

The results also showed that it is possible to distinguish voltammetrically if a specific sequence of known content in guanine is forming self-dimers and intra-strand structures in the ODN lipoplex. If the value of peak-dG current is found to be significantly below that expected from its guanine content (which can be determined easily through calibration) this reflects the fact that not all guanine residues are accessible to undergo oxidation, and can be used as a preliminary experimental screening (or alert) for the presence of interor/and intra-strand duplex structures.

In conclusion, the results presented demonstrate the potential use of voltammetric techniques to study and characterise physicochemical properties of ODN lipoplexes.

\section{Acknowledgements}

Financial support from Fundação para a Ciência e Tecnologia (FCT), Ph.D. Grants PRAXIS XXI/SFRH/BD/6134/ 
2001 (JAPP) and PRAXIS XXI/SFRH/BD/4897/2001 (MM), POCTI (co-financed by the European Community Fund FEDER), ICEMS (Research Unit 103) and European Projects QLK3-2000-01311 and ERBICT15CT960804 are gratefully acknowledged.

\section{References}

Alessenko, A.V., Burlakova, E.B., 2002. Functional role of phospholipids in the nuclear events. Bioelectrochemistry 58, 13-21.

Audory, S., Hoekstra, D., 2001. Cationic lipid-mediated transfection in vitro and in vivo (review). Mol. Membr. Biol. 18, 129-143.

Bordi, F., Cametti, C., Gili, T., Gaudino, D., Sennato, S., 2003. Time evolution of the formation of different size cationic liposome-polyelectrolyte complexes. Bioelectrochemistry 59, 99-106.

Brett, C.M.A., Oliveira-Brett, A.M., 1998. Electroanalysis. Oxford University Press, New York.

Choosakoonkriang, S., Wiethoff, C.M., Koe, G.S., Anchordoquy, T.J., Middaugh, C.R., 2003. An infrared spectroscopy study of the effect of hydration on cationic lipid/DNA complexes. J. Pharm. Sci. 92, $115-130$.

Cohen, J.S., 1994. Gene-mimetic substances: drugs designed to intervene in gene expression. Adv. Pharmacol. 25, 319-339.

Dunlap, D.D., Maggi, A., Soria, M.R., Monaco, L., 1997. Nanoscopic structure of DNA condensed for gene delivery. Nucl. Acids Res. 25, 3095-3101.

Even-Chen, S., Barenholz, Y., 2000. DOTAP cationic liposomes prefer relaxed over supercoiled plasmids. Biochim. Biophys. Acta 1509, 176-188.

Hianik, T., Vitovic, P., Humenik, D., Andreev, S.Y.U., Oretskaya, T.S., Hall, E.A.H., Vadgama, P., 2003. Hybridization of DNA at the surface of phospholipid monolayers. Effect of orientation of oligonucleotide chains. Bioelectrochemistry 59, 35-40.

Hong-Ping, D., Kwok-Keung, S., 1996. Voltammetric studies of electrochemical pretreatment of rotating-disc glassy carbon electrode in phosphate buffer. J. Electroanal. Chem. 419, 7-14.

Jurkiewicz, P., Okruszek, A., Hof, M., Langner, M., 2003. Associating oligonucleotides with positively charged liposomes. Cell Mol. Biol. Lett. 8, 77-84.

Kuvichkin, V.V., 2002. DNA-lipid interactions in vitro and in vivo. Bioelectrochemistry $58,3-12$.

Meidan, V.M., Cohen, J.S., Amariglio, N., Hirsch-Lerner, D., Barenholz, Y., 2000. Interaction of oligonucleotides with cationic lipids: the relationship between electrostatics, hydration and state of aggregation. Biochim. Biophys. Acta 1464, 251-261.

Meidan, V.M., Glezer, J., Amariglio, N., Cohen, J.S., Barenholz, Y., 2001. Oligonucleotide lipoplexes: the influence of oligonucleotide composition on complexation. Biochim. Biophys. Acta 1568, 177182.

Monkkonen, J., Urtti, A., 1998. Lipid fusion in oligonucleotide and gene delivery with cationic lipids. Adv. Drug Del. Rev. 34, 37-49.

Nikolelis, D.P., Hianik, T., Krull, U.J., 1999. Biosensors based on thin lipids films and liposomes. Electroanalysis 11, 7-15.

Oliveira-Brett, A.M., Matysik, F.-M., 1997. Voltametric and sonovoltametric studies on the oxidation of thymine and cytosine at a glassy carbon electrode. J. Electroanal. Chem. 429, 95-99.

Oliveira-Brett, A.M., Serrano, S.H.P., Piedade, J.A.P., 1999. Electrochemistry of DNA. In: Compton, R.G., Hancock, G. (Eds.), Comprehensive Chemical Kinetics, vol. 37. Elsevier, Amsterdam, Chapter 3, pp. 91-119.

Palecek, E., Fojta, M., Jelen, F., Vetterl, V., 2002. Electrochemical analysis of nucleic acids. In: Bard, A.J., Stratmann, M. (Eds.), The Encyclopaedia of Electrochemistry, vol. 9: Bioelectrochemistry. Wiley/VCH Verlag, Weinheim, Federal Republic of Germany, pp. 365-429.

Pedroso de Lima, M.C., Simões, S., Pires, P., Faneca, H., Düzgünes, N., 2001. Cationic lipid-DNA complexes in gene delivery: from biophysics to biological applications. Adv. Drug Del. 47, 277-294.

Piedade, J.A.P., Mano, M., Pedroso de Lima, M.C., Oretskaya, T.S., Oliveira-Brett, A.M., 2004. Voltammetric behaviour of oligonucleotide lipoplexes adsorbed onto glassy carbon electrodes. J. Electroanal. Chem. 564, 25-34.

Prasad, T.K., Gopal, V., Rao, N.M., 2003. Structural changes in DNA mediated by cationic lipids alter in vitro transcriptional activity at low charge ratios. Biochim. Biophys. Acta 1619, 59-69.

Raedler, J., Koltover, I., Salditt, T., Safinya, C., 1997. Structure of DNA-cationic liposome complexes: DNA intercalation in multilamellar membranes in distinct interhelical packing regimes. Science 275, 810-814.

Ruponen, M., Honkakoski, P., Rönkkö, S., Pelkonen, J., Tammi, M., Urtti, A., 2003. Extracellular and intracellular barriers in non-viral gene delivery. J. Contr. Release 93 (2), 213-217.

Simões, S., Slepushkin, V., Gaspar, R., Pedroso de Lima, M.C., Düzgünes, N., 1998. Gene delivery by negatively charged ternary complexes of DNA, cationic liposomes and transferrin or fusigenic peptides. Gene Ther. 5, 955-964.

Siontorou, C.G., Oliveira-Brett, A.M., Nikolelis, D.P., 1996. Evaluation of a glassy carbon electrode modified by a bilayer lipid membrane with incorporated DNA. Talanta 43, 1137-1144.

Wang, J., 2000. From DNA biosensors to gene chips. Nucl. Acids Res. 28, 3011-3016.

Weimin, H., Zheling, Z., Xiaojun, H., Jilin, T., Jianguo, W., Shaojun, D., Erkang, W., 2003. Liposome-mediated conformation transition of DNA detected by molecular probe: methyl green. Bioelectrochemistry 59, 21-27.

Wiethoff, C.M., Gill, M.L., Koe, G.S., Koe, J.G., Middaugh, C.R., 2002 The structural organization of cationic lipid-DNA complexes. J. Biol. Chem. 277, 44980-44987. 